\title{
Genetic Recombination in the Hop-Wilt Fungus Verticillium albo-atrum
}

\author{
By A. C. HASTIE \\ Rothamsted Experimental Station, Harpenden, Hertfordshire
}

(Received 13 October 1960)

\begin{abstract}
SUMMARY
Heterokaryons obtained between nutritional mutants of the imperfect fungus Verticillium albo-atrum (Reinke \& Berth) were very unstable, and heterokaryotic hyphal tips growing out from mixed inocula were difficult to find. Heterokaryotic conidia were not detected, in spite of the fact that about $1 \%$ of the spores contained two or three nuclei. Heterozygous nuclei in uninucleate conidia from mixed cultures yielded recombinant phenotypes at a higher frequency than usually observed in the other imperfect fungi which have been studied genetically. With no easily applicable criterion of chromosome number, such as spore size, the interpretation of results is difficult, but the genetic recombination demonstrated here seems likely to be accomplished through a parasexual system. Nuclei heterozygous at some loci but homozygous at others were formed from nuclei heterozygous at four marked loci, and were detected by isolating second-order segregants. These heterozygous segregants were presumably produced by mitotic recombination.
\end{abstract}

\section{INTRODUCTION}

The history of Verticillium wilt of hops follows a familiar pattern. The initial outbreaks reported by Harris (1927) were mild, but more virulent strains of the fungus were reported about 10 years later (Keyworth, 1939). Severe attacks by Verticillium have become more frequent and wilted plants of a hitherto wilttolerant variety were recently reported. It is therefore of interest to know whether genetic recombination may be a factor in the evolution of these more virulent strains. Genetic recombination has been reported in several fungi which, like Verticillium albo-atrum, have no known sexual stage (Pontecorvo, Roper \& Forbes, 1953; Pontecorvo \& Sermonti, 1954; Buxton 1956). Robinson, Larson \& Walker (1957) suggested genetic recombination as a probable explanation of phenomena observed in their monoconidial cultures of $V$. albo-atrum, which appears to be a convenient subject for genetical investigations since the conidia are almost always uninucleate (Waggoner, 1956; Caroselli, 1957). The establishment of heterokaryons should present no difficulties, because Reinke \& Berthold (1879) reported anastomosing germ tubes, and Caroselli (1957) showed that some of the hyphal cells contain several nuclei.

\footnotetext{
* Present address: Botany Dept., Queen's College, Dundee.
} 


\section{METHODS}

Dark and hyaline variants of Verticillium albo-atrum (Reinke \& Berth) isolated from wilted hop plants at East Malling Research Station, Kent, were used. The dark strains produced black torulose hyphae, not formed by the hyaline isolates. These were formed only irregularly on most media, but an augmented prune extract (PE) medium (Talboys, 1960) was found to be suitable for distinguishing between dark and hyaline strains.

Conidia of Verticillium albo-atrum are borne on verticillately branched conidiophores, and a drop of water containing up to thirty conidia is formed at the tip of each branch. Suspensions of conidia were readily prepared by flooding young slope cultures with a few millilitres of sterile distilled water. Hyphal fragments occurred only rarely in these suspensions, and the conidia did not clump as do those of dryspored fungi when suspensions are prepared in this way. Thus it was reasonably certain that the colonies formed when aliquots of these suspensions were diluted and spread on agar media originated from single conidia. Isolations of single conidia were made at certain stages during the work. For this, a conidial suspension was spread on the surface of agar media and the conidia, which are about $7 \mu$ long, were located under high magnification and isolated on small blocks of agar with the aid of a dissecting microscope. The nuclei in conidia were counted after staining with Giemsa. The staining schedule suggested by Hrushovetz (1956) was satisfactory, but shortening the hydrolysis time to $4 \mathrm{~min}$. at $60^{\circ}$ gave better results.

Stable nutritional mutants were obtained by exposing conidia to ultra-violet radiation supplied by a Hanovia XII medium-pressure lamp from which about $95 \%$ of the radiation was emitted at $2537 \AA$. Conidia were exposed at $20 \mathrm{~cm}$. from the ultraviolet source, at which distance the radiation intensity was about $108 \mu \mathrm{W} / \mathrm{cm} .{ }^{2}$. An exposure of $20 \mathrm{sec}$. gave only $3 \%$ survivors, of which $0.5 \%$ were nutritional mutants.

Wild type strains of the fungus grew rapidly on Czapek-Dox agar and this was used as a minimal medium. Complete medium (CM medium; Pontecorvo, 1953) was also extensively used, and the techniques of replication and auxanography described by Pontecorvo were used to characterize nutritional mutants. Heterokaryons were synthesized by mixing conidia of nutritional mutants, and streaking the mixed inoculum across the diameter of Petri dishes containing Czapek-Dox agar medium. Conidia containing heterozygous nuclei were selected by the technique of Roper (1952).

A spontaneous mutant resistant to acriflavine was selected by inoculating about: $180 \times 10^{6}$ conidia on to $\mathrm{CM}$ medium containing $100 \mu \mathrm{g}$. acriflavine $/ \mathrm{ml}$. Acriflavine tolerance was determined on CM medium containing an appropriate concentration (300 $\mu \mathrm{g} . / \mathrm{ml}$.) of acriflavine added to the agar medium immediately before it was poured into Petri dishes. This procedure gave consistent results despite the interaction between acriflavine and nucleic acid noted by McIlwain (1941), and there was no obvious lag phase during the early stages in growth of the resistant mutant.

The following symbols are used for convenient reference: $a d=$ adenineless; $b i=$ biotinless $;$ meth $=$ methionineless $;$ acr $=$ acriflavine-resistant. 


\section{RESULTS}

\section{Heterokaryosis}

Conidia of a hyaline, methionineless and biotinless (meth bi) strain were mixed with conidia of a black adenineless $(a d)$ isolate. The mixed inoculum, incubated on unaugmented Czapek-Dox agar on which neither strain can grow, produced fans of mycelium which outgrew the mixed inoculum, although growing more slowly than the wild type (Pl. 1, fig. 1). Most hyphal tips taken from these sectors failed to grow on Czapek-Dox agar medium, but eight hyphal tips from three sectors formed cultures which later yielded conidia of both parental types. Such cultures were clearly heterokaryotic. Because only parental type conidia were detected in samples of about 1000 conidia from each of these heterokaryons, it appeared that heterokaryotic conidia were produced very infrequently.

The intensity of the black phenotype varied more among colonies produced by 200 adenineless conidia from the heterokaryons than in a similar sample of conidia taken directly from the original pure culture. Because of this variability the mycelial colour will not be mentioned in the section on genetic recombination but will be considered further in the discussion.

\section{Selection of prototrophic conidia}

A spontaneous mutant resistant to acriflavine was selected from the methionineless and biotinless strain used to investigate heterokaryosis. This further marker gave the strain the phenotype acr meth bi. Conidia from this strain were mixed with conidia from the black adenineless acriflavine-sensitive isolate previously used, and the mixed inoculum was incubated on slopes of Czapek-Dox agar medium. Similar samples of conidia from these mixed cultures were spread on Czapek-Dox, and

\section{Table 1. Numbers of prototrophic conidia recovered from five heterokaryons of Venticillium albo-atrum}

Heterokaryotic mycelium was selected by incubating two auxotrophic strains on Czapek-Dox agar (acr meth $b i \times a d)$. Conidial suspensions prepared from these heterokaryons were then spread on Czapek-Dox agar and Czapek-Dox + acriflavine to select prototrophs and acriflavine-resistant prototrophs, respectively.

$\begin{array}{cccc}\text { Heterokaryons } & \begin{array}{c}\text { No. of conidia } \\ \text { tested on each } \\ \text { medium }\left(\times \mathbf{1 0}^{6}\right)\end{array} & \text { Czapek-Dox } & \begin{array}{c}\text { Media } \\ \text { Czapek-Dox } \\ \text { Acriflavine }\end{array} \\ \text { CF 1 } & 10.8 & 3 & 0 \\ \text { CF2 } & 12.5 & 8 & 0 \\ \text { CF 4 } & 18.3 & 9 & 0 \\ \text { CF5 } & 16.4 & 10 & 0 \\ \text { CF 10 } & 10.5 & 3 & 0 \\ \text { Total } & 68.5 & 33 & 0\end{array}$

Czapek-Dox containing $100 \mu \mathrm{g} . / \mathrm{ml}$. acriflavine, and the number of colonies formed after 4 days' incubation are recorded in Table 1 . The recovery rate was approximately one prototrophic conidium per two million tested, and the test also indicated that these prototrophic conidia were acriflavine-sensitive. To confirm that the prototrophic propagules were uninucleate conidia, suspensions of conidia were 
prepared from four of the colonies recorded in Table 1, and aliquots of these suspensions stained with Giemsa. About $99 \%$ of the conidia in each of these four suspensions were uninucleate, and of about 4000 conidia examined none had more than three nuclei. Other samples of the same suspensions were spread at low density on both CM medium and Czapek-Dox medium to estimate the proportion of prototrophs. None of the four suspensions contained less than $30 \%$ prototrophic conidia. Because there were very few, if any, non-viable conidia it must be concluded that almost all the prototrophic conidia were uninucleate. The analysis of heterokaryons indicated that multinucleate conidia must be homokaryotic.

\section{Recovery of recombinant phenotypes}

Single conidia isolated from one of the suspensions referred to above were incubated on slopes of Czapek-Dox medium, and only the prototrophs retained. Conidia from the resulting cultures harvested after incubation for 10 days were spread on CM medium at a density of about thirty per Petri dish. The colonies formed were re-isolated in a regular pattern for replication on an approximate series of test media (Pl. 1, fig. 2), and the phenotypes of about 100 isolates from each of twenty monoconidial cultures are given in Table 2. It is quite certain that each of the twenty cultures analysed was produced by a single conidium, which was likely to be uninucleate or at least homokaryotic, and the phenotypes recovered show that these conidia must have contained nuclei heterozygous for the four markers. There are therefore sixteen possible phenotypes obtainable from each culture, and in Table 2 these are arranged in reciprocal pairs.

The frequencies with which any two phenotypes were recovered can be compared by estimating the mean difference in the numbers of observations over the twenty replicates, and obtaining a ' $t$ ' test from the ratio of this mean difference to its standard error. The parental phenotypes acr meth $b i+$, and $+++a d$ were recovered most frequently, and the recovery rates did not differ significantly ( $P=0 \cdot 3$ to $0 \cdot 2)$. This is also true of the reciprocal phenotypes $a c r+b i a d$ and + meth $++(P=\mathbf{0 . 3}$ to $\mathbf{0} \cdot 2$ ), and it can similarly be shown that each of these pairs was recovered in significantly different numbers $(P<\mathbf{0 . 0 0 1})$. There is therefore a tendency for reciprocal pairs to be recovered equally frequently although the phenotypes acr +++ and + meth bi ad are an obvious exception to this since 469 of the former were found, but only one of the latter. This difference can be accounted for by clonal multiplication of the original heterozygous nucleus in each culture.

\section{The isolation of second-order segregants}

Conidial dimensions provide a convenient distinction between haploid and diploid recombinants in some imperfect fungi shown to have a parasexual genetic system (Pontecorvo, 1956). Unfortunately the frequency distribution of the conidial dimensions in cultures of the parental strains used here did not differ significantly from other cultures known to have originated from a single heterozygous conidium.

The twenty cultures analysed in Table 2 were produced by conidia containing nuclei heterozygous at all four loci, and if the segregants were formed by parasexual mechanisms (haploidization and mitotic recombination) we should expect some of the first-order isolates recorded in Table 2 to be haploid and the others diploid. 


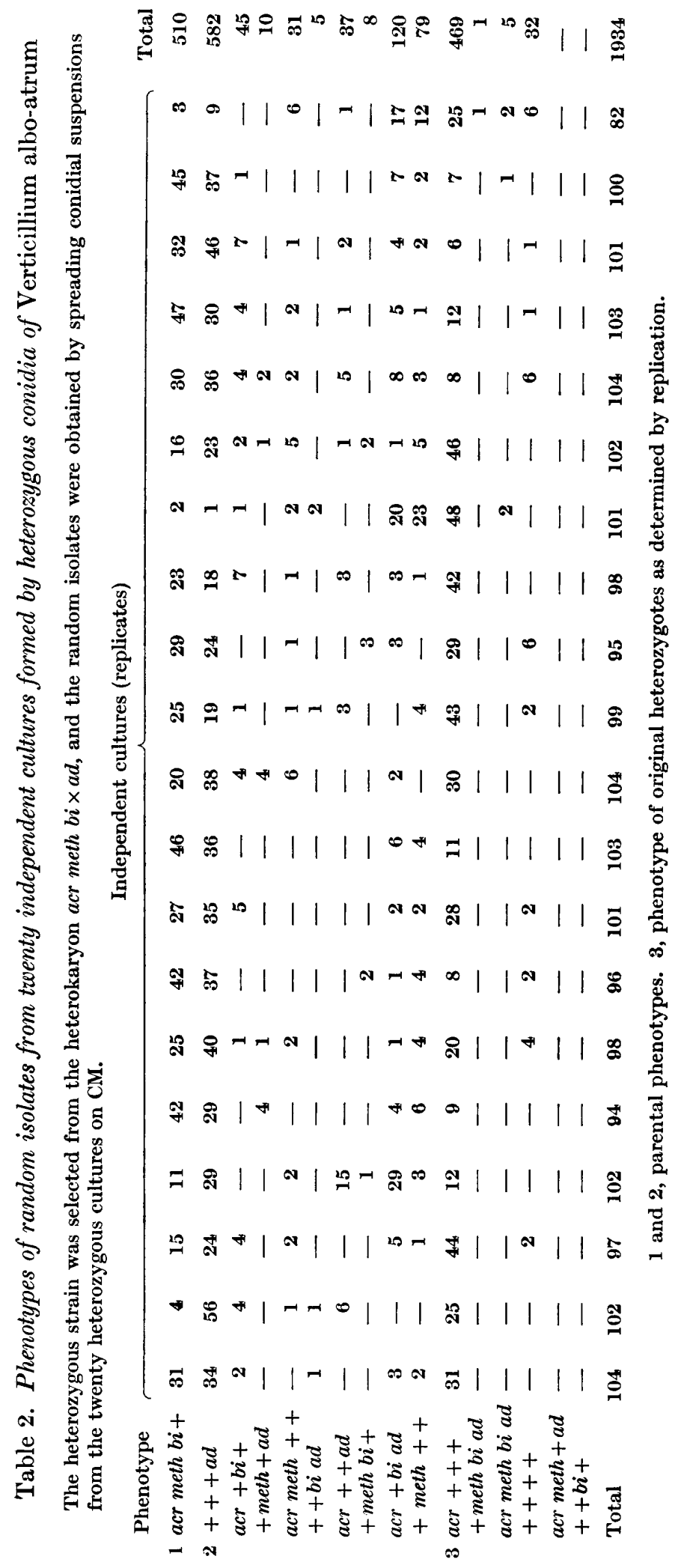


The diploid first-order segregants would be homozygous for some markers, and heterozygous at the other loci, and would therefore be detected by their capacity to produce further segregant phenotypes (second-order segregants), as shown in Aspergillus nidulans by Pontecorvo, Tarr-Gloor \& Forbes (1954).

The very high yield of recombinant phenotypes from the 10-day-old 'mosaic' cultures, segregating at all four loci, suggested that heterozygous first-order segregant genotypes may be readily detected in random samples of conidia from these cultures, but they were not. However, when 'mosaic' cultures, segregating at all four loci, were grown on an augmented prune extract medium, the colonies were slightly slower growing and more fluffy than pure cultures. Thus it seems that secondorder segregants may be recovered from colonies with this morphology which also have nutritional deficiency or are sensitive to acriflavine (i.e. fluffy first-order segregants). Four colonies with these characters were noticed during the later stages of the work, and two of them are shown in Pl. 1, figs. 3 and 4. Mono-conidial isolates were made from each of these and Table 3 gives the classification of approximately 100 isolates from each purified culture. Plate 1, fig. 5, shows the phenotypes recovered in a sample of twenty-six random isolates from a heterozygous segregant culture.

Table 3. Phenotypes of second-order isolates from four heterozygous segregant cultures of Verticillium albo-atrum

The heterozygous segregants were recovered amongst random first-order isolates
from the heterozygote acr meth bi/ad, and their probable genotypes inferred from the characterization of second-order isolates.

Phenotypes of second-order isolates

$$
+++
$$$$
+ \text { meth }++
$$$$
++a d
$$

+ meth $+a d$

+ meth $b i+$

$++b i+*$

acr meth $b i+$

acr meth ++
Phenotypes of the heterozygous segregant cultures

$\begin{array}{cccc}++++ & ++++ & ++++ & \text { acr meth }++ \\ 33 & 19 & 75 & - \\ 24 & 39 & 2 & 48 \\ 39 & 43 & 9 & - \\ - & 3 & 10 & - \\ - & - & 4 & - \\ - & - & - & 36 \\ - & - & - & 10\end{array}$

Probable genotypes of the original heterozygous segregant nuclei

$$
\begin{array}{cccc}
+ \text { meth }++ & + \text { meth }++ & + \text { meth } b i+ & \text { acr meth } b i+ \\
+++a d & +++a d & +++a d & + \text { meth }++
\end{array}
$$

* This phenotype was not observed among random first-order isolates (Table 2).

The four loci segregated at approximately the same frequency in the cultures reported in Table 2 and if the conidia which initiated the cultures recorded in Table 3 also contained nuclei heterozygous at all four loci we should again expect all loci to show similar segregation rates. The column on the left of Table 3 shows that culture to be segregating at only two loci (meth and $a d$ ), and the proportion of non-segregants at each of these loci is approximately $2 / 3$. This means that the probability of any single conidium taken at random from this culture being, say, methionine independent is $2 / 3$. The chance of getting ninety-six meth + spores from this culture in that many trials is then $(2 / 3)^{96}$. As the biotin locus can be expected to segregate at a similar frequency when it is heterozygous, the probability of the 
original nucleus being heterozygous $b i /+$ is therefore about $(2 / 3)^{96}$, and there is even less chance of the nucleus being heterozygous at both the acriflavine and biotin loci. This reasoning has also been applied to the other three cultures recorded in Table 3, and the genotypes suggested by the observed segregations are given at the bottom of that Table. The single conidia from which the cultures were grown could not have been merely heterokaryotic, rather than heterozygous, as the isolates made from each culture included a high proportion which were fluffy like the original culture, had the same phenotype, and which showed similar segregation patterns.

Attempts to select rare acriflavine-resistant recombinants from the sensitives shown in Table 3 failed. This gives further support to the suggestion that the original conidia contained nuclei homozygous, or hemizygous, at the non-segregating loci. The genotypes suggested for these nuclei at the bottom of Table 3 assume they were diploid but there is no proof of this in the absence of a convenient criterion of chromosome number. Loci showing segregation must be heterozygous, but other loci in the same nucleus which do not segregate may be either haploid (hemizygous) or homozygous and diploid. These nuclei could arise by either the loss of one or more chromosomes from the diploid set, or by mitotic crossing-over, and there is no way of distinguishing between these possibilities using only the present data.

\section{DISCUSSION}

Variation in wild-type cultures of Verticillium albo-atrum has been described by Waggoner (1956), Caroselli (1957), Robinson et al. (1957), and Van den Ende (1958). Their cultures usually produced black torulose hyphae but variants apparently unable to do so were often observed. All forty single conidia which Robinson et al. (1957) took from a hyaline monospore culture produced cultures with black hyphae, and they suggested this was probably a result of genetic recombination. It seems at least equally likely that the original hyaline culture was potentially black (i.e. had the appropriate genotype) but failed to develop this phenotype; environmental factors such as $\mathrm{pH}$, temperature, carbohydrate source, presence of alcohols, and even the depth of the medium, can all greatly influence the development and colour of these hyphae.

The doubly auxotrophic parent (acr meth $b i+$ ) used in my work never formed black hyphae on any of the media used, but the adenineless parent always did to some extent when grown on augmented prune extract. The analysis of heterokaryons between these strains did not convincingly demonstrate that the black character was entirely controlled by the nucleus. Further, nuclei heterozygous for all the selected markers always formed colonies with black hyphae, and no isolate from these colonies had the hyaline phenotype of the respective parent, although a few recombinants were obtained which formed black hyphae only rarely. The existing evidence suggests that cytoplasmic factors have a dominant role in the expression, and perhaps the inheritance, of the mycelial colour. Colour was certainly not inherited as a single gene difference otherwise it would have segregated at a similar rate to the nutritional markers. There is certainly no reason to suppose that the hyaline variants often isolated from wild-type cultures of Verticillium albo-atrum always arise by genetic recombination.

The most striking feature of the genetic recombination reported here is the rela- 
tively high frequency at which it occurs. No attempt was made to estimate the absolute frequency with which recombinant phenotypes were produced, but it must certainly be greater than that usually observed in the other imperfect fungi previously studied (Pontecorvo et al. 1953; Pontecorvo \& Sermonti, 1954; Buxton, 1956). Relatively high rates of recombination induced by mutagens have been reported in Aspergillus orzyae (Ikeda, Ishitani \& Nakamura, 1956), Penicillium chrysogenum (Morpurgo \& Sermonti, 1959), and Aspergillus nidulans (Käfer, 1960).

The high frequency of recombination leads to an anomaly seen by comparing Tables 1 and 2. The results in Table 1 indicate that the heterozygous prototrophic conidia selected directly from the heterokaryon were acriflavine-sensitive, but heterozygotes of the same genotype were classified as acriflavine-resistant in Table 2 for which the conidia were characterized by replication as opposed to direct-plating (Table 1). By replication we seek to classify a conidium by replicating the colony which it produces, and therein assume a relatively pure culture was formed. The discrepancy between the results in Tables 1 and 2 may be resolved by assuming acriflavine-resistance is recessive to sensitivity. Single heterozygous conidia $(a c r /+)$ tested by direct-plating will then be acriflavine sensitive (Table 1), but because the novel genotypes acr and acr/acr arise frequently during growth of a colony they may be transferred to the test medium in replicating, and the isolate will consequently appear acriflavine-resistant although it was in fact formed by an acriflavinesensitive conidium. Corresponding misclassification cannot arise with respect to the nutritional markers, and the high frequency of segregation and recombination remains even if the acriflavine marker is ignored.

The genetic system in Verticillium albo-atrum seems likely to be mitotic rather than meiotic, and the question arises whether the high rate of recombination reported here is usual in $V$. albo-atrum or results from an irregular chromosome complement in the heterozygote. Analysis of the colonies produced by thirtythrec prototrophic conidia selected directly from eight mixed cultures of the two parental strains gave results similar to those recorded in Table 2 , and this regularity suggests that the very unstable heterozygous nuclei are those formed initially by nuclear fusions. If one of the parent strains had a disomic chromosome number (say $n+1$ ), selection within this strain should be expected to favour a haploid genotype, and heterozygous diploid nuclei rather than trisomics (say $2 n+1$ ) would usually be formed in mixed cultures by nuclear fusions between the complementary parental genotypes. It is therefore unlikely that one parent strain with an irregular chromosome number would consistently lead to the production of heterozygotes with a correspondingly abnormal chromosome number, and that this could cause the high frequency of recombination.

A clirumosomal rearrangement, or structural change, involving a centromere in one of the parents, so that non-disjunction or loss of one chromosome is frequent, could explain the results obtained. Loss of one chromosome from some nuclei in a haploid strain would go undetected because the nullisomic nuclei formed would be eliminated, but if the same chromosome was lost from a diploid nucleus this would result in monosomic nuclei which would not be eliminated, but tend to give further segregation. Before deciding whether the high frequency of recombination observed here is usual in Verticillium albo-atrum, more heterozygotes from independently isolated wild-type cultures will have to be analysed. 
In Table 2 there is a tendency for the pairs of reciprocal phenotypes to be recovered equally frequently, and each pair at a significantly different rate with the parental classes most frequent. This pattern clearly suggests the four markers are linked, but elucidation of the formal genetics of this heterozygote requires that the haploid genotypes included in the data in Table 2 be considered separately.

The work described in this paper was done during the tenure of a Research Studentship awarded by the Agricultural Research Council (A.R.C.). I thank the A.R.C. for their support, Dr E. W. Buxton, Rothamsted Experimental Station, for his helpful suggestions, and Professor G. Pontecorvo, F.R.S., University of Glasgow, for his criticisms of the original draft. I also wish to acknowledge my thanks to Mr F. Cowland, Rothamsted Experimental Station, for taking the photographs.

\section{REFERENCES}

Buxton, A. W. (1956). Heterokaryosis and parasexual recombination in pathogenic strains of Fusarium oxysporum. J. gen. Microbiol. 15, 133.

Caroseldi, N. E. (1957). Verticillium wilt of maple. Bull. R. I. agric. Exp. Sta. 335.

Harris, R. V. (1927). A wilt disease of hops. Rep. E. Malling Res. Sta. for 1925.

Hrushovetz, S. B. (1956). Cytological studies in Helminthosporium sativum. Canad. J. Bot. 34, 321.

Ikeda, Y., Ishitani, C. \& Nakamura, K. (1956). A high frequency of heterozygous diploids and somatic recombination induced in imperfect fungi by ultra violet light. Proc, int. Genet, Symp. p. 547.

KäFER, E. (1960). High frequency of spontaneous and induced somatic segregation in Aspergillus nidulans. Nature, Lond. 186, 619.

KeYworth, W. G. (1939). Verticillium wilt of hops. Rep. E. Malling Res. Sta. for 1938, 244.

MCILwaIN, H. (1941). A nutritional investigation of the anti-bacterial action of acriflavine. Biochem. J. 35, 1311.

Morpurgo, G. \& Sermontr, G. (1959). Chemically induced instability in a heterozygous diploid of Penicillium chrysogenum. Genetics, 44, 137.

Ponteconvo, G. (1953). The genetics of Aspergillus nidulans. Advanc. Genet. 5, 141.

Ponteconvo, G. (1956). The parasexual cycle in fungi. Annu. Rev. Microbiol. 10, 393.

Ponteconvo, G., Roper, J. A. \& Forbes, E. (1953). Genetic recombination without sexual reproduction in Aspergillus niger. J. gen. Microbiol. 8, 198.

Pontecorvo, G. \& Sermonti, G. (1954). Parasexual recombination in Penicillium chrysogenum. J. gen. Microbiol. 11, 94.

Pontecorvo, G., Tarr-Gloor, E. \& Forbes, E. (1954). Analysis of mitotic recombination in Aspergillus nidulans. J. Genet. 52, 226.

Reinke, J. \& Berthold, G. (1879). Die Zersetzung der Kartoffel durch Pilze. Untersuchungen aus dem Botanischen Laboratorium der Universität Göttingen, $1,1$.

Robinson, D. B., Larson, R. H. \& Walker, J. C. (1957). Verticillium wilt of potato in relation to epidemiology, symptoms and variability of the pathogen. Univ. Wis. Res. Bull. p. 202.

Roper, J. A. (1952). Production of heterozygous diploids in filamentous fungi. Experimentia, 8, 14.

Talboys, P. W. (1960). A culture-medium aiding the identification of Verticillium alboatrum and V. Dahliae. Plant Path. 9, 57.

VAN DEN ENde, G. (1958). Untersuchungen über den Pflanzenparasiten Verticillium albo-atrum. Acta bot. neerl. 7, 665.

WAGGONER, P. E. (1956). Variation in Verticillium albo-atrum from potato. Plant Dis. Reptr, 40, 429. 


\section{EXPLANATION OF PLATE}

Fig. 1. Pure and mixed inocula of auxotrophs on Czapek-Dox agar. Adenineless strain (left), methionineless and biotinless strain (right), and mixed inoculum producing heterokaryotic sectors. (centre).

Fig, 2. Random monoconidial isolates from a heterozygous monoconidial culture. Template cultures grown on PE medium (top right) and replicates of this template on CM medium containing $300 \mu \mathrm{g} / \mathrm{ml}$. acriflavine (lower right), biotinless medium (top left), methionineless medium (midleft), and adenineless medium (bottom left) $\left(\times \frac{1}{2}\right)$.

Fig. 3. Two fluffy (heterozygous) isolates in twenty-six random monoconidial isolates from a segregating culture (PE medium).

Fig. 4. Reverse view of the isolates shown in Fig. 3 to show sectoring associated with fluffy phenotypes (PE medium).

Fig. 5. Random monoconidial isolates from a heterozygous monoconidial segregant culture. Template cultures grown on PE medium (top right), and replicates of this template on CM medium containing $300 \mu \mathrm{g} / \mathrm{ml}$. acriflavine (lower right), methionineless medium (top left), biotinless medium (mid left), and adenineless medium (bottom left) $\left(\times \frac{1}{2}\right)$. 

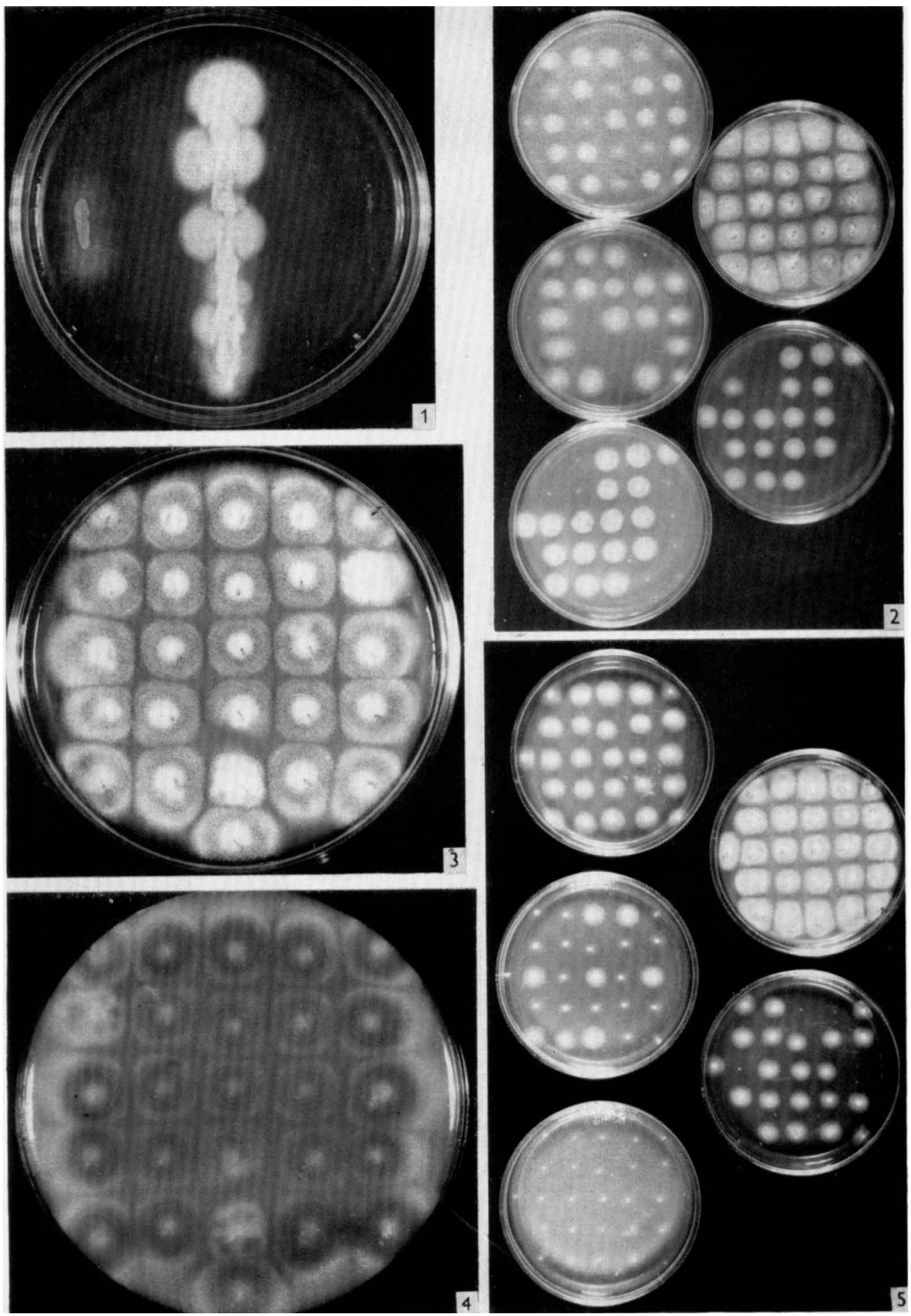

A. C. HASTIE

(F'acing p. 38:) 